\title{
LEDs for Thermographic NDT: Status and Chances
}

\author{
by Mathias Ziegler, Henrik Steinfurth, Mathias Röllig, Philipp Myrach, and Christiane Maierhofer
}

BAM Federal Institute for Materials Research and Testing, Division 8.4, Unter den Eichen 87, 12205 Berlin, Germany, mathias.ziegler@bam.de

\begin{abstract}
Light emitting diodes (LEDs) allow for a defined high-speed modulation of high optical power density without interfering with the thermal radiation answer of the test specimen. We present the current status of kilowatt class highpower LED application in active thermography and highlight chances concerning their application in alternative and novel testing techniques.
\end{abstract}

\section{Introduction}

Application of optical energy sources in thermographic NDT offers the outstanding advantage of being an entirely contact free and remote NDT technique. The most common light sources flash lamps and incandescent lamps are relatively cheap while offering high power densities at the testing site. Usually the modulation frequency of incandescent lamps is low $(<1 \mathrm{~Hz})$ and the rise and fall times are relatively high $(>100 \mathrm{~ms})$ whereas flash lamps deliver short pulses $(<10 \mathrm{~ms})$ at low repetition rates $(>1 \mathrm{~Hz})$ only. LEDs might fill this gap. They exhibit a rapid evolution concerning achievable power density making them more and more interesting for thermographic NDT application. LEDs allow for modulation frequencies beyond $100 \mathrm{~Hz}$ and thereby for thermal penetration depths below $1 \mathrm{~mm}$. Hence they potentially offer applications in lockin-thermography of metals and in near surface testing of layered systems, both in a more controllable and quantitative fashion than presently performed using standard light sources. Similarly to lasers, LEDs feature first, a narrow spectral distribution of their radiation power and, second, a spectral distribution that can be designed to meet the maximal absorption of the tested material, both at moderate costs. Based upon electroluminescence, LEDs generate a low portion of thermal radiation only which allows thermographic testing while the sample is heated. In contrast, flash and incandescent lamps need spectral filtering of a significant portion of their intrinsically generated thermal radiation in order to reduce interferences with the "true" thermal answer emitted by the heated sample.

We present the current status of kilowatt class high-power LED application in active thermography and highlight chances concerning their application in alternative and novel testing techniques. For example, we are now able to use nearly arbitrary temporal excitation functions since LEDs feature a virtually lag-free emission of full power. It is expected that this new degree of freedom will result in better and more exact penetration depth and depth resolution capabilities compared to classical techniques using either single frequency lockin or powerful short pulse excitation. 